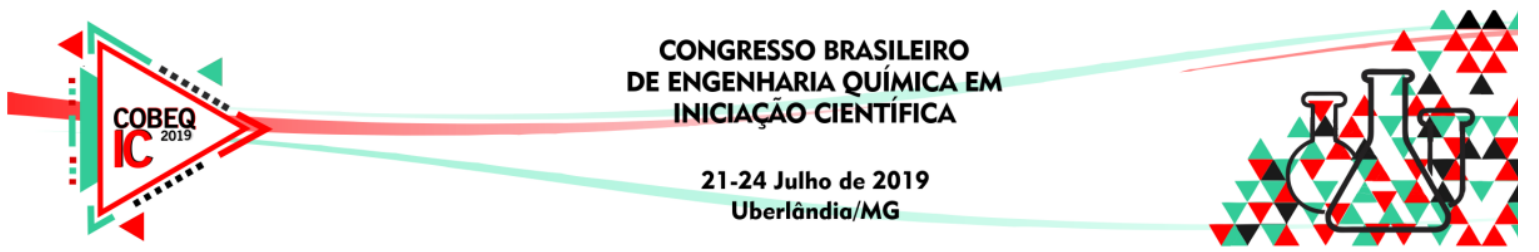

\title{
MODELAGEM E SIMULAÇÃO DA PRODUÇÃO DE ÉTERES ETÍLICOS DE ETILENOGLIÓIS EM MEIO NÃO CATALÍTICO E CATALÍTICO UTILIZANDO BICABORNATO (HCO3-)
}

\author{
H. M. GUERREIRO ${ }^{1}$ e E. Q. FONSECA JÚNIOR ${ }^{1,2}$ \\ ${ }^{1}$ Universidade Federal do Amazonas, Departamento de Engenharia Química \\ ${ }^{2}$ Universidade do Estado do Amazonas, Programa de Pós-Graduação em Biotecnologia e \\ Recursos Naturais da Amazônia \\ E-mail para contato: engenhariaquimicahg@gmail.com
}

\begin{abstract}
RESUMO - A Oxiteno desempenha uma produção anual de éteres mistos do etilenoglicol com 60.000 toneladas, diante desses dados é cada vez mais importante a busca pela otimização desses processos. A partir disso, o presente trabalho tem como objetivo realizar simulações no COCO 3.3 das reações de produção de EEMEG, EEDEG e EETEG visando determinar uma maior seletividade de EEDEG em meio não catalítico e catalítico utilizando 0,15 mol de HCO3-/L através da variação de parâmetros operacionais e físico-químicos. O planejamento fatorial apontou que a temperatura de entrada e o comprimento do reator PFR estão diretamente relacionados, evitando assim modificações operacionais. O meio catalítico apresentou uma baixa seletividade do EEDEG por favorecer a reação de EEMEG. Diante disso, obteve-se o EEDEG em 22,65 \% utilizando a temperatura de $145{ }^{\circ} \mathrm{C}$ num reator PFR de $40 \mathrm{~m}^{2}$ adiabático a $35 \mathrm{kgf} / \mathrm{cm}^{2} \mathrm{em}$ meio não catalítico e partindo de duas colunas de destilação de 5 estágios para separação dos produtos.
\end{abstract}

\section{INTRODUÇÃO}

Os etilenoglicóis apresentam interesse econômico no mercado global como o monoetilenoglicol com consumo de 15 milhões de toneladas anuais (Hal et al., 2007), diante disso a otimização dessa área, minimizando gastos e aumentando a pureza dos produtos tem sua importância. Na América Latina, a Oxiteno é líder na produção de tensoativos e especialidades químicas com a produção anual de 60.000 (sessenta mil) toneladas de éteres mistos do etilenoglicol e 310.000 (trezentos e dez mil) toneladas do monômero e oligômeros do etilenoglicol (Martins e Cardoso, 2005). Em 2019 a empresa lançou um desafio no Programa de Pós-graduação em Engenharia de Processos Químicos e Bioquímicos (EPQB) da UFRJ com o objetivo de otimizar a produção de éter etílico de dietilenoglicol (EEDEG) nas condições especificadas pela empresa. Nesse desafio, a empresa informa que a sua porcentagem de produção atual é de 66,6 \% de éter etílico de monoetilenoglicol (EEMEG), 24,9 \% de EEDEG, $7,9 \%$ de éter etílico de trietilenoglicol (EETEG) e 0,6 \% de pesados. Tais produtos são obtidos da reação de etanol com óxido de etileno utilizando hidróxido de potássio $(\mathrm{KOH})$ como catalisador. Dentre esses produtos destaca-se o EEDEG, utilizado principalmente na produção de fluido de freios, que exige alta pureza e parâmetros físico-químicos bem definidos justificando a importância de sua produção (Martins e Cardoso, 2005). 


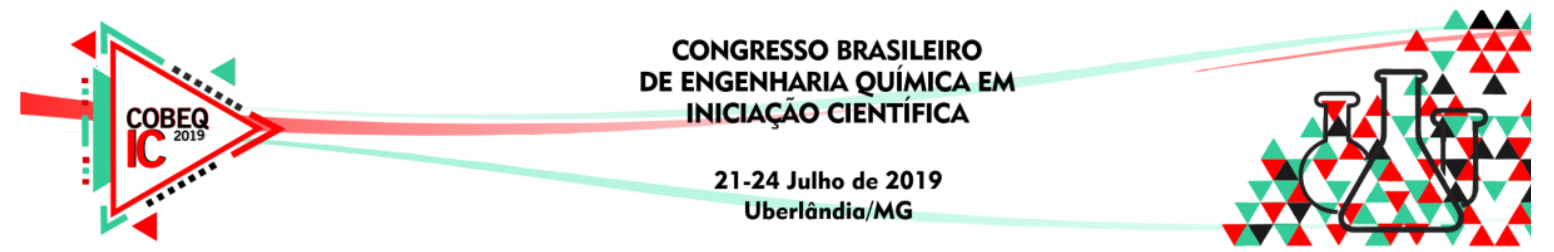

Como ferramenta, a simulação computacional se tornou prática e objetiva, evitando gastos com experimentos e servindo de base para estudos posteriores, onde sua utilização serve também de suporte em estudos de novos processos químicos em aproximações coerentes. Em função disso, o presente trabalho tem como objetivo estudar a produção desses compostos, por meio de planejamento $2^{3}$, em meio não catalítico e catalítico utilizando bicarbonato (HCO3-), a fim de avaliar a produção e a seletividade do EEDEG em diminuição ao do EEMEG utilizando condições operacionais pré-estabelecidas pela empresa Oxiteno.

\section{MATERIAIS E MÉTODOS}

\subsection{Simulador e Dados Cinéticos}

O simulador utilizado foi o COCO 3.3, que permite a simulação de processos químicos em estado estacionário através de métodos numéricos convencionais. Foi utilizado o software Statistica 7.0 para a análise das interações entre as variáveis e elaboração dos gráficos.

Os dados cinéticos, utilizados neste trabalho, para simular o processo de produção dos éteres etílicos de etilenoglicóis, foi desenvolvido por Altiokka e Akyalçin (2009), que utilizaram da reação de água e óxido de etileno para produzir monoetilenoglicol (MEG), dietilenoglicol (DEG) e trietilenoglicol (TEG) em meio não catalítico e catalítico utilizando bicabornato (HCO3-).

Segundo Martins e Cardoso (2005), o pH do reagente utilizado influencia diretamente na seletividade dos produtos de etilenoglicóis, o que pode ser observado na Tabela 1 os compostos hidroxilados e seus $\mathrm{pKa}$ 's.

Tabela 1 - Compostos hidroxilados e seus respectivos pKa's

\begin{tabular}{|c|c|}
\hline Composto & $\mathrm{pKa}$ \\
\hline \hline Fenol & 9,90 \\
\hline MEG & 14,22 \\
\hline EEMEG & $\sim 14,50^{*}$ \\
\hline Metanol & 15,00 \\
\hline Água & 15,70 \\
\hline Etanol & 16,00 \\
\hline
\end{tabular}

* Valor aproximado

Observa-se na Tabela 1 que a água e o etanol apresentam valores muito próximos de pKa, com isso as equações da cinética de Altiokka e Akyalçin (2009) foram utilizadas como uma aproximação na produção de EEMEG, EEDEG e EETEG através da reação do óxido de etileno com etanol, tanto para o meio não catalítico quanto para o catalítico utilizando o mesmo catalisador de estudo.

As equações segundo Altiokka e Akyalçin (2009) são, para meio não catalítico:

$$
\begin{aligned}
& k_{1} /[L /(\mathrm{mol} \cdot \min )]=\exp (13.62-8220 / T) \\
& k_{2} /[L /(\mathrm{mol} \cdot \min )]=\exp (15.57-8700 / T)
\end{aligned}
$$




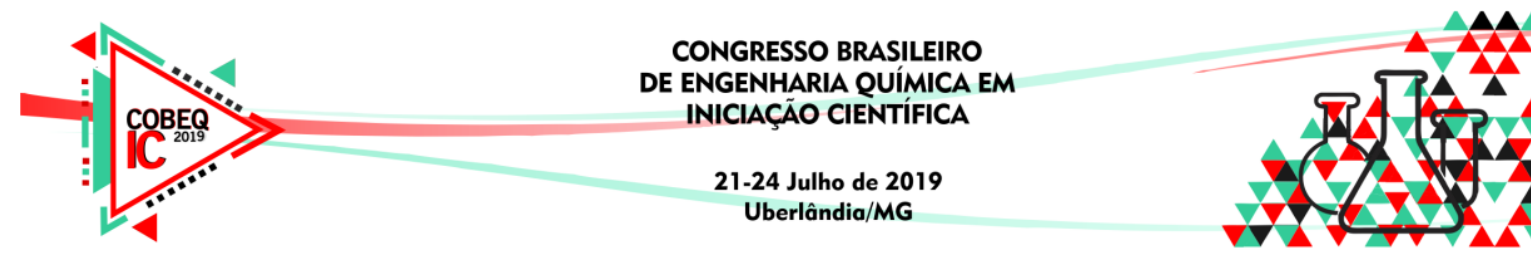

$k_{3} /[L /(\mathrm{mol} \cdot \mathrm{min})]=\exp (16.06-8900 / T)$

E para meio catalítico utilizando $0,15 \mathrm{~mol}$ de $\mathrm{HCO}^{-} / \mathrm{L}$ :

$$
\begin{gathered}
k_{1} /[\mathrm{L} /(\mathrm{mol} \cdot \mathrm{min})]=\exp (19.60-10446 / T) \\
k_{2} /[\mathrm{L} /(\mathrm{mol} \cdot \mathrm{min})]=\exp (20.19-10421 / \mathrm{T}) \\
k_{3} /[\mathrm{L} /(\mathrm{mol} \cdot \mathrm{min})]=\exp (19.06-10018 / T)
\end{gathered}
$$

Sendo a temperatura em Kelvin.

\subsection{Modelo Experimental}

Segundo a empresa Oxiteno, a produção dos éteres etílicos de etilenoglicóis corresponde a faixa de temperatura entre $135{ }^{\circ} \mathrm{C}$ e $145^{\circ} \mathrm{C}$, na pressão de $35 \mathrm{kgf} / \mathrm{cm}^{2} \mathrm{em}$ um reator PFR adiabático com uma relação Etanol/Óxido de Etileno igual a 3,6. E ainda segundo Martins e Cardoso (2005), os métodos de separação empregados para esses produtos são realizados por colunas de destilação. Dessa forma, foi montado o flowsheet descrito na Figura 1 e por meio dos estudos de Ferreira (2016) e Chvidchenko (2008) optou-se pelo modelo termodinâmico de Peng Robinson para a produção dos éteres etílicos de etilenoglicóis.

Figura 1 - Planta para produção de éteres etílicos de etilenoglicóis

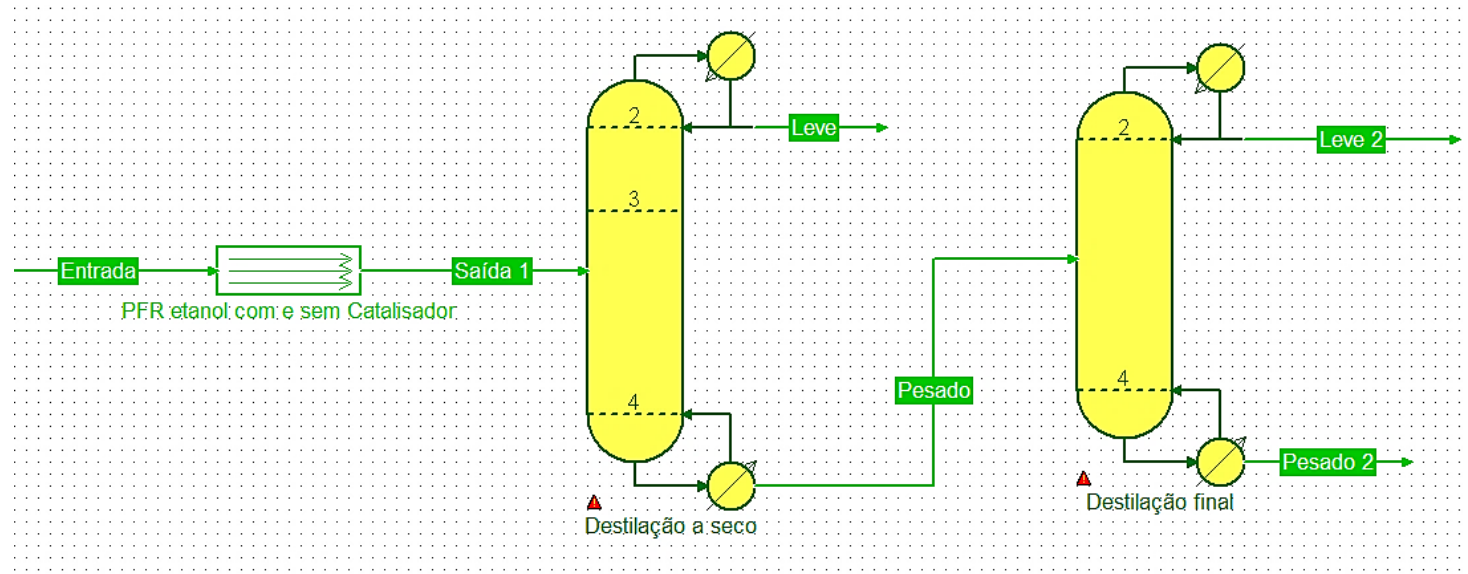

Para as análises manteve-se fixo os valores de pressão no reator a $35 \mathrm{kgf} / \mathrm{cm}^{2} \operatorname{com} 2 \mathrm{~m}$ de diâmetro e nas colunas de destilação a pressão de $1 \mathrm{~atm}$, onde cada coluna possui 5 (cinco) estágios para a separação dos produtos.

Para a investigação dos resultados, foi realizado um planejamento fatorial $2^{3}$, sendo os fatores avaliados: temperatura de alimentação (A), comprimento do reator (B) e catalisador (C) através dos pontos apresentados na Tabela 2, selecionados após testes apontarem que depois de um dado volume, dependendo da temperatura de entrada e do catalisador, a reação se torna plenamente desenvolvida e portanto o aumento do volume do reator não é mais significativo. 


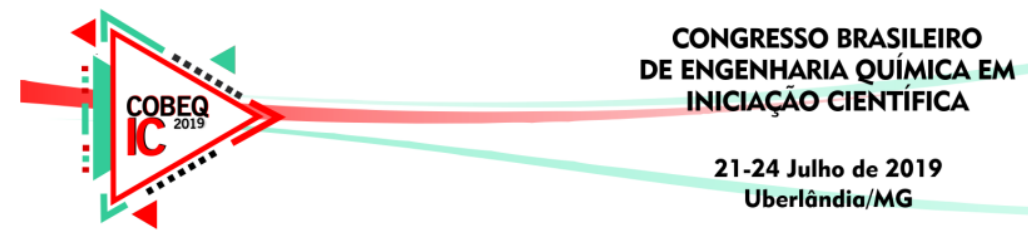

Tabela 2 - Dados do planejamento fatorial $2^{3}$ utilizados na simulação

\begin{tabular}{|c|c|c|}
\hline Variável & Nível -1 & Nível +1 \\
\hline \hline $\mathrm{A}\left({ }^{\circ} \mathrm{C}\right)$ & 135 & 145 \\
\hline $\mathrm{B}(\mathrm{m})$ & 10 & 20 \\
\hline $\mathrm{C}$ & Sem catalisador & Com catalisador \\
\hline
\end{tabular}

\section{RESULTADOS E DISCUSSÃO}

O planejamento fatorial apresentou a Tabela 3 com informações de interação entre os fatores:

Tabela 3 - Análise dos resultados do planejamento fatorial

\begin{tabular}{|c|c|c|c|}
\hline Fator & Efeito Estimado & $\begin{array}{c}\text { Soma dos } \\
\text { Quadrados }\end{array}$ & $\begin{array}{c}\text { Contribuição } \\
\text { Percentual }\end{array}$ \\
\hline \hline $\mathrm{A}$ & 0,4125 & 0,680625 & 5,10490126774 \\
\hline $\mathrm{B}$ & 0,5725 & 1,311025 & 9,83309926103 \\
\hline $\mathrm{C}$ & 0,4175 & 0,697225 & 5,2294064814 \\
\hline $\mathrm{AB}$ & $-0,3975$ & 0,632025 & 4,74038600366 \\
\hline $\mathrm{AC}$ & $-0,8925$ & 3,186225 & 23,8976882157 \\
\hline $\mathrm{BC}$ & $-1,0025$ & 4,020025 & 30,151450092 \\
\hline $\mathrm{ABC}$ & 0,8375 & 2,805625 & 21,0430686785 \\
\hline
\end{tabular}

A Tabela 3 mostra que a maior contribuição percentual vem da interação entre B e C que são comprimento e catalisador. A interação entre a temperatura e o catalisador também se mostrou bastante evidente assim como a interação entre os três fatores, isso mostra que os fatores agindo isoladamente contribuem muito pouco para melhoria dos resultados.

Observou-se ainda que a variação do terceiro parâmetro no processo não seria necessária, pois a contribuição percentual da interação entre A e B foi a menor de todas, com isso observa-se que não é interessante que o comprimento e a temperatura sejam variados no mesmo experimento, pois sua contribuição é pouco significante ao processo o que fica exposto no diagrama de pareto da Figura 2. Portanto é mais interessante a variação somente do catalisador e do comprimento, ou temperatura, visto que os melhores resultados são oriundos principalmente dessas.

Figura 2 - Resultado do planejamento pelo Diagrama de Pareto

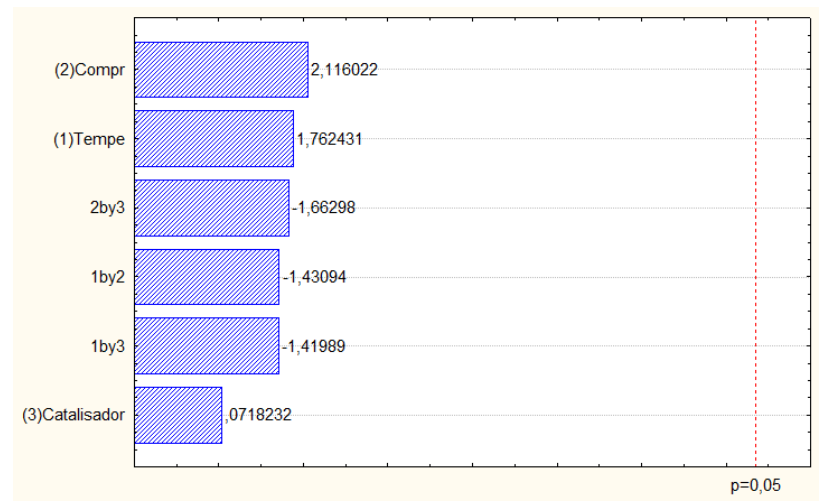




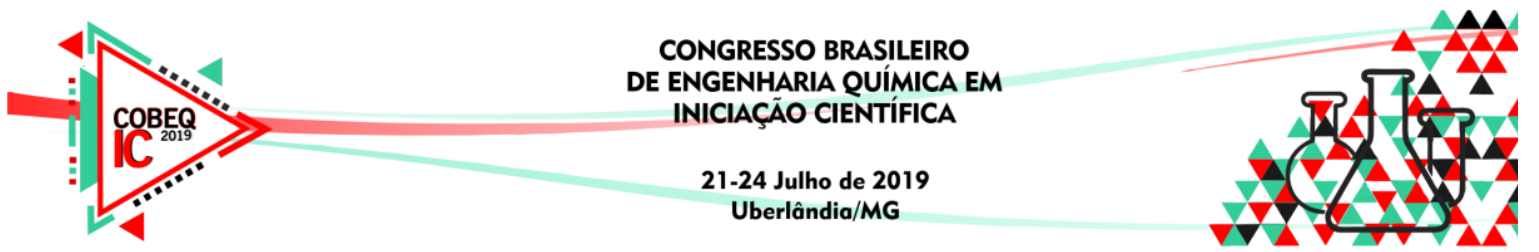

Como mostra o diagrama de pareto (Figura 2), a interação entre comprimento ou temperatura com catalisador se mostrou com influência negativa, visto que o catalisador não superou a seletividade encontrada no meio não catalítico, ou seja, independente da sua interação com temperatura ou comprimento, o catalisador não corresponderá resultados satisfatórios dentro da faixa de dados estabelecida indicando que melhores resultados poderão ser obtidos por meio não catalítico.

Em função disso, avaliou-se as equações cinéticas das reações e então observou-se que o meio catalítico desfavoreceu a seletividade do EEDEG, pois o meio não catalítico apresentou valores ascendentes no sentido da reação e por causa desse comportamento o meio catalítico favoreceu a primeira, por possuir os valores mais altos de velocidade de reação, enquanto que no meio não catalítico as últimas reações é que foram favorecidas.

Diante disso percebeu-se que no meio não catalítico a seletividade do EEMEG tende a diminuir, pois na saída, sem e com catalisador, os fluxos de EEDEG foram próximos enquanto que a de EEMEG decaí sem o catalisador, ou seja, a seletividade do EEMEG é inversamente proporcional ao EEDEG em semelhança com os estudos da relação de MEG e DEG feitos por Hal et al. (2007), ao relatarem que quando o meio catalítico não possui alta seletividade em relação ao MEG a seletividade do DEG e glicóis maiores aumentariam.

O diagrama de pareto (Figura 2) também apontou que os fatores comprimento e temperatura agindo isoladamente estavam apresentando comportamento afins, o que indicou uma relação direta entre os dois, e em relação a interação entre comprimento e temperatura o gráfico reafirmou o fato de a influência ser negativa em concordância com a contribuição percentual da Tabela 3 .

Em relação a variação somente da temperatura e comprimento, que apresentaram valores afins de influência no processo, o gráfico de superfície de resposta (Figura 3) apresentou os resultados do sistema com o meio não catalítico onde percebeu-se que os melhores resultados são provenientes de alta temperatura e comprimento, com isso determinou-se que o meio não catalítico se mostrou mais sensível às mudanças de temperatura de alimentação e comprimento do reator, justamente porque no meio catalítico a reação se desenvolve mais rapidamente, exigindo menos volume dependendo da temperatura de entrada.

Figura 3 - Gráfico de superfície da interação entre comprimento e temperatura

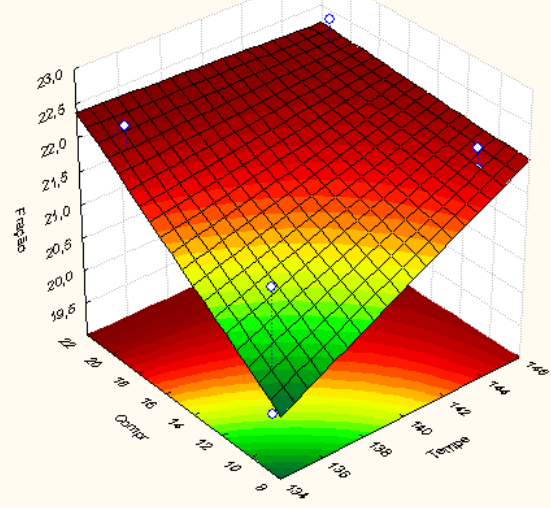




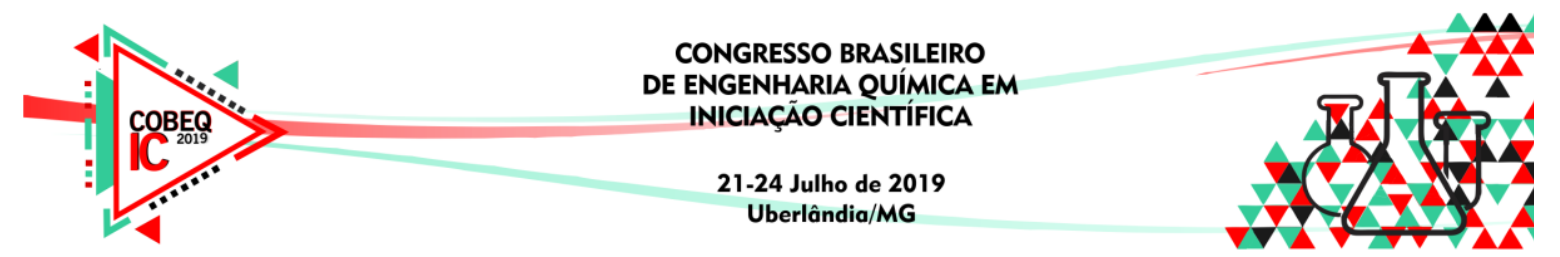

Em função disso, o melhor resultado encontrado foi no meio não catalítico na temperatura de $145{ }^{\circ} \mathrm{C}$ e comprimento do PFR em $20 \mathrm{~m}$ apresentando uma seletividade do EEDEG de 22,65 \%. No meio catalítico, as variações se apresentaram mínimas em relação a modificação de temperatura e comprimento do reator, onde sua faixa de resultados possui um mínimo de $21,38 \%$ e máximo de $21,78 \%$, enquanto que no meio não catalítico o mínimo é de $19,50 \%$ justificando o comportamento do gráfico da Figura 3.

\section{CONCLUSÃO}

A simulação e o planejamento fatorial proporcionaram dados e informações desejadas nesta investigação, onde se observou que a seletividade de EEDEG se mostrou sensível as modificações físico-químicas e operacionais do meio e se apresentou mais elevada em condições não catalíticas e ainda foi evidenciado que os fatores comprimento e temperatura estão relacionados diretamente por causa da afinidade entre o tempo de reação e temperatura no reator PFR o que permite a viabilidade do processo evitando modificações nos equipamentos e alterando somente parâmetros físico-químicos.

\section{REFERÊNCIAS}

ALTIOKKA, M. R.; AKYALÇIN, S. Kinetics of the hydration of ethylene oxide in the presence of heterogeneous catalyst. Ind. Engin. Chem. Resea., v. 48, n. 24, p. 1084010844, 2009.

CHVIDCHENKO, V. Estudo comparativo do desempenho das equações de estado CPA, SRK e PR na modelagem de sistemas de interesse para a indústria do gás natural. Dissertação de Mestrado. Univer. Fed. Rio de Jan. 2008

FERREIRA, P. C. Avaliação de desempenho de modelos termodinâmicos para sistemas a altas pressões contendo $\mathrm{CO}_{2}$. Dissertação de Mestrado. Univer. Fed. Rio de Jan.2016.

HAL, J. W.; LEDFORD, J. S.; ZHANG, X. Investigation of three types of catalysts for the hydration of ethylene oxide (EO) to monoethylene glycol (MEG). Catal. Today, v. 123, n. 1-4, p. 310-315, 2007.

MARTINS, L.; CARDOSO D. Produção de etilenoglicóis e derivados por reações catalíticas do óxido de eteno. Quím. Nov., v. 28, n. 2, p. 264-273, 2005.

OXITENO. Desafio EPQB / Empresas: Desafio 1. Disponível em < http://tpqb.eq.ufrj.br/ download/desafio-epqb-empresas-1.pdf $>$. 